\title{
Improved Post-Resuscitation Survival Time with Adjuvant Cytosolic Energy Replenishment in a Murine Model of Hemorrhagic Refractory Shock
}

\author{
El Rasheid Zakaria ${ }^{*}$, Bellal Joseph ${ }^{1}$, Faisal S Jehan ${ }^{1}$, Muhammad Khan ${ }^{1}$, Abdelrahman Algamal ${ }^{2}$, Faheem Sartaj ${ }^{2}$, Muhammad Jaffar Khan ${ }^{3}$ and Rajvir Singh $^{3}$ \\ ${ }^{1}$ Division of Trauma, Critical Care, Burns \& Emergency Surgery, The University of Arizona, Tucson, AZ 85721, USA \\ ${ }^{2}$ Biomedical Research Center \& College of Arts and Health Sciences, Qatar University, P.O. Box 2713, Doha, Qatar \\ ${ }^{3}$ Hamad Medical Corporation, P.O. Box 3050, Doha, Qatar
}

*Corresponding author: El Rasheid Zakaria, Division of Trauma, Critical Care, Burns \& Emergency Surgery, The University of Arizona, Tucson, AZ 85721, USA, Tel: +520 626-9010; Fax: +520 626 5016; E-mail: drelzak@surgery.arizona.edu

Received Date: Sep 26, 2017; Accepted Date: Nov 16, 2017; Published Date: Nov 20, 2017

Copyright: $\odot 2017$ Zakaria ER, et al. This is an open-access article distributed under the terms of the Creative Commons Attribution License, which permits unrestricted use, distribution, and reproduction in any medium, provided the original author and source are credited.

\begin{abstract}
Objective: A refractory haemorrhagic hypovolemic shock (HS) resuscitation is challenging. HS is associated with profound depletions of cellular energy nucleotides that can cause death from a cardio-circulatory arrest. To prevent an imminent cardio-circulatory arrest, vasopressors, commonly norepinephrine is usually temporarily administered to manage a persistent hypotension that is not corrected by aggressive resuscitation efforts. The objective of this study is to determine the post-resuscitation survival time after adjuvant resuscitations of a refractory HS with norepinephrine, vasopressin or direct cytosolic energy (adenosine-5 -triphosphate, ATP) replenishment using lipid vesicles encapsulating ATP (ATPv).
\end{abstract}

Methods: 50 male Sprague-Dawley rats were randomized to 5 groups of 10 each: HS/conventional resuscitation (CR), HS/CR+Norepinephrine, HS/CR+Vasopressin, HS/CR+Vesicles, and HS/CR+ATPv. (HS=initial removal of $30 \%$ of the calculated blood volume, a 60 min hypotensive phase, and a subsequent transection of the spleen for uncontrolled haemorrhage until persistent shock index $(\mathrm{SI})>5$ and mean arterial pressure $(\mathrm{MAP})<35 \mathrm{mmHg}$ were achieved; $\mathrm{CR}=$ shed blood returned+double the shed blood volume as lactated Ringer's solution). Direct cytosolic ATP replenishment was accomplished with ATPv, which are highly fusogenic lipid vesicles encapsulating ATP. Fusion of the ATPv with the cell membrane on contact, allows for direct cytosolic ATP delivery. We determined the post-resuscitation survival time as the end-point of the study.

Results: All animals displayed the same class of shock as demonstrated by the SI and MAP. Median postresuscitation survival times (computed by the Kaplan-Meier survival curves and the long-rank Mantel-Cox test) were as follows: $\mathrm{HS} / \mathrm{CR}=35.5 \mathrm{~min} ; \mathrm{HS} / \mathrm{CR}+$ Norepinephrine=38.5 min; HS/CR+Vasopressin=20 min; HS/CR+Lipid Vesicles control=88.5 $\mathrm{min}$; and $\mathrm{HS} / \mathrm{CR}+\mathrm{ATPV}=158.5 \mathrm{~min}(\mathrm{p}<0.001)$.

Conclusion: The replenishment of the depleted cellular cytosolic energy stores in a refractory haemorrhagic hypotensive shock prolongs post-resuscitation survival time and delays cardio-circulatory arrest. This buys time for the initiation of definitive resuscitation protocols. Cellular energy failure appears to contribute to the pathogenesis of shock refractoriness to resuscitation efforts. The temporary administration of vasopressors for pressure-support resuscitation of a refractory haemorrhagic hypovolemic shock exerts no survival benefits.

Keywords: Haemorrhagic shock; Resuscitation; Cellular energy; Vasopressors; Cytosolic energy delivery

\section{Introduction}

Trauma remains one of the most common causes of mortality and morbidity worldwide [1]. Exsanguinating haemorrhage and the resultant shock is the most common cause of mortality in the first hour of arrival to a trauma centre and accounts for almost half of the trauma deaths in the first $24 \mathrm{~h}[2,3]$. The consensus in the management of traumatic haemorrhage entails rapid homeostasis and subsequent correction of the volume deficit $[4,5]$. The most recent human data have confirmed that correction of the volume deficit by balanced transfusions of plasma, packed red blood cells, and platelets in a 1:1:1 ratio achieved homeostasis and improved the $24 \mathrm{~h}$ survival as compared with the traditional methods of resuscitation including crystalloids [6-8]. Nevertheless, despite these modern resuscitation protocols, the management of a refractory shock that is associated with prolonged hypotension remains a challenge. In such a situation, vasopressors, commonly norepinephrine, is added to the resuscitation protocol to manage a persistent hypotension that is not responsive to massive transfusions; and to protect against an imminent cardiocirculatory collapse [9]. Today, there is no prospective evidence to support the efficacy vasopressors in improving resuscitation outcome after a refractory shock. It is likely that the refractoriness of shock to resuscitation efforts is attributed, at least in part, to the failures to maintain adequate cellular energy levels and end-organ perfusion to satisfy the energy-dependent cellular processes and the tissue metabolic demands. 
The role of cellular energy failure in the pathogenesis of traumatic haemorrhage is well established [10-13]. Studies in animals have demonstrated profound depletions of cellular levels of adenosine nucleotides after traumatic haemorrhage [14,15]. This occurs as the cellular energy production mechanisms shift from the aerobic high energy-yield to the anaerobic glycolysis. Similarly, our systematic studies with dual-label quantitative autoradiography have demonstrated depletions of the local intravascular volume in all organs even after adequate resuscitation that restored and maintained central hemodynamic [16]. This result occurs as the trans-capillary starling forces, which maintain a balanced intravascular volume, are perturbed by both haemorrhagic shock and by resuscitation in favour of a rapid trans-capillary fluid escape rate and depletion of the intravascular volume. However, attempts to correct this energy failure by the administration of exogenous magnesium-containing nucleotides like adenosine-5'-triphosphate (ATP) have been unsuccessful. This is attributed to two limiting effects: 1) ATP cannot pass through the cell membrane in quantities sufficient to replenish and subsequently satisfy cellular and tissue metabolic requirements $[17,18]$; and 2) the half-life of free ATP in the blood is less than $40 \mathrm{~s}$, limiting its efficacy as a bioenergetics substrate. However, these limitations have been resolved by the development of ATPv, which is highly fusogenic Nano-lipid vesicle encapsulating ATP. ATPv fuses with cell membranes on contact, thereby delivers its ATP load directly into the cytosol of cells [19].

The continued depletion of the cellular energy nucleotides together with the contracted local intravascular volume contribute to the failure to maintain adequate end-organ perfusion and hence, irreversibility of shock. To examine this reasoning, we sought to determine the effect of direct cytosolic energy replenishment with ATPv on the postresuscitation survival time in a rat model of lethal refractory shock. We hypothesized that in a refractory shock, the adjuvant use of ATPv along with conventional resuscitation improves post-resuscitation survival time as compared with conventional resuscitation alone or pressure-support resuscitation with either adjuvants norepinephrine or vasopressin to conventional resuscitation.

\section{Methods}

\section{General animal preparation}

The Institutional Animal Care and Use Committees (IACUC) of Hamad Medical Corporation and Qatar University (Doha, Qatar) preapproved the experimental protocols. All experiments were performed on male Sprague Dawley rats (weighing 200-220 g) procured from Harlan Laboratories (Correzzana, Italy). They were fed standard rat chow and allowed free access to water and feeding until the day of the experiment. On that day, anesthesia was induced with an intraperitoneal injection of $60 \mathrm{mg} / \mathrm{kg}$ of Pentobarbitone (Troy Laboratories Pty Ltd, Plumpton, Australia), which was maintained during the course of the experiment with a supplemental subcutaneous injection of $25 \%$ of the induction dose at $60 \mathrm{~min}$ intervals. Body temperature was maintained at $37^{\circ} \pm 0.5^{\circ} \mathrm{C}$ with a rectal probe and a servo-controlled heating pad (TC-1000, Temperature Controller, CWE Incorporated, Ardmore PA, USA). We carried out surgery after the loss of blink and withdrawal reflexes. A tracheostomy was performed to reduce airway resistance and to allow for spontaneous room-air breathing. We cannulated the left carotid artery and then the right femoral vein with PE-50 tubing for continuous blood pressure recording using a calibrated pressure transducer connected to a blood pressure analyser (BPA-400, Digi-Med, Louisville, KY, USA), and for blood withdrawal and resuscitation, respectively. A mid-line laparotomy was performed for access to the spleen, which was transected for the induction of uncontrolled haemorrhage, according to protocol. During the course of the experiment, at 5 min intervals, we recorded the mean arterial pressure (MAP); and calculated the Shock index (SI) as the ratio of heart rate to systolic pressure.

\section{Characterization of the model of refractory haemorrhagic shock}

We performed our study in a reproducible rat model of lethal refractory haemorrhagic hypovolemic shock. Our model encompasses significant tissue damage (abdominal laparotomy incision, transections of the splenic parenchyma, and a vascular injury), a reproducible class of refractory shock, and inherent $100 \%$ mortality without resuscitation intervention. We induced a refractory haemorrhagic hypovolemic shock in two phases of haemorrhage separated by $60 \mathrm{~min}$ hypovolemia. Phase-1 involved pump-controlled venous withdrawal of $30 \%$ of the animal's calculated blood volume over 15 min using the formula of Lee and Blaufox [20]. Hypovolemia was then maintained for $60 \mathrm{~min}$, followed by a phase- 2 of uncontrolled haemorrhage as induced by transections of the splenic parenchyma at the two ends of the organ as well as the severing of one of the branches of the splenic artery. The transected organ was returned to the abdominal cavity for free arterial and venous bleeding until a preset point for homeostasis and resuscitation intervention was reached. This was defined by the following: 1) the need to infuse $0.5 \mathrm{ml}$ saline to restore the mean arterial pressure (MAP) to $50 \mathrm{mmHg}$ at two consecutive occasions of precipitous blood pressure drops within a $10 \mathrm{~min}$ period during the phase of uncontrolled haemorrhage; and, 2) a rapid increase and sustainability of the SI to a value $>5$ during the phase of uncontrolled haemorrhage [21]. In our model, the two criteria that triggered the initiation of resuscitation were reached within 10-15 min after the splenic injury in all animals. The time of animal death was defined by a pulse-less, zero mean arterial pressure on the continued hemodynamic digital recording together with no respiration effort.

\section{Chemical, drugs and tissue-bath solution}

All chemicals were purchased from the Sigma Chemical Company (St. Louis, Missouri, USA). We obtained Levophed (Norepinephrine Bitartrate, Hospira Inc., Lake Forest, IL, USA) and Pitressin (Synthetic Vasopressin, Par Pharmaceutical, Inc., Parsippany, NJ, USA) from Hamad Medical Center Pharmacy. The energy delivery vehicle (ATPv) was purchased from Energy Delivery Solutions (Energy Delivery Solutions, LLC, Jeffersonville, IN, USA). We added the designated vasopressor (or ATPv) to the crystalloid resuscitation fluid according to protocol. ATPv is a special formulation of Adenosine-5'triphosphate (ATP) encapsulated in nanoscale delivery vehicles (lipid vesicles), which are highly fusogenic. Fusion of the lipid vesicles with the cell membrane and the rate of delivery of ATP directly into the cytosol of cells have all been verified and validated by the manufacturer in human umbilical endothelial cells, rat hepatocytes, and rat cardiomyocytes.

\section{Experimental protocol}

In execution of the experimental protocol depicted in Figure 1, we enrolled fifty animals in our study. 


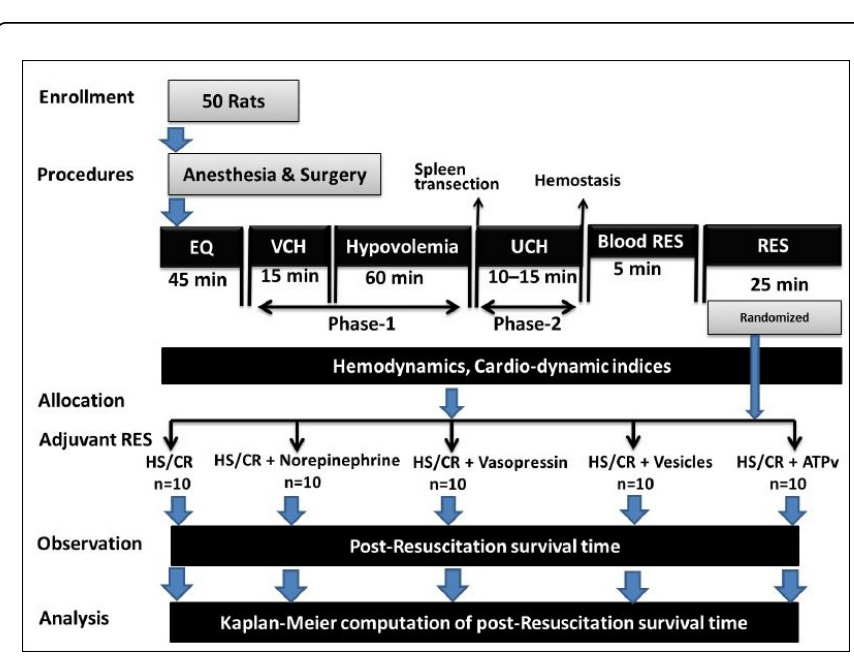

Figure 1: Six phases (Enrollment, Procedures, Allocation, Intervention, Observation and Data Analysis) of a randomized trial of five experimental resuscitation groups. EQ=Equilibration Phase; $\mathrm{VCH}=$ Volume Controlled Haemorrhage $(30 \%$ of the estimated blood volume); UHS=Uncontrolled Haemorrhage; Blood RES=Return of the Shed Blood Over $5 \mathrm{~min}$; HS=Haemorrhagic Shock; $\mathrm{CR}=$ Conventional Resuscitation (return of shed blood over $5 \mathrm{~min}$ followed by $2 \mathrm{x}$ the shed blood volume as ringers lactate infused over the subsequent $25 \mathrm{~min}$ ). All adjuvants were admixed with the ringers lactate crystalloid solution; Lipid Vesicles=Lipid Vesicles (no ATP); and ATPv=Lipid Vesicles Encapsulating ATP; ATP $=$ Adenosine $-5^{`}{ }^{\prime}$ Triphosphate.

After induction of anesthesia, all animals received a subcutaneous injection of $2 \mathrm{ml}$ of warmed normal saline to compensate for any fluid loss during animal preparation. Following the surgical procedures, there was a stabilization period of $45 \mathrm{~min}$ to allow the animal to recover from surgical stress. We took the baseline hemodynamic measurements during the last $10 \mathrm{~min}$ of the equilibration period. Then, all animals underwent the first phase of a volume-controlled haemorrhage. This was initiated by pump-controlled withdrawal (Genie Touch, Kent Scientific Corporation, Torrington, CT, USA) of venous blood (femoral vein), totalling $30 \%$ of the animal's estimated blood volume over $15 \mathrm{~min}$. Following protocol, the shed blood was preserved in a pre-rinsed heparin-coated tube (Heparin Leo, $1000 \mathrm{IU}$ per ml, Ballerup, Denmark) for later re-infusion. All animals stayed hypovolemic during the subsequent $60 \mathrm{~min}$. At the end of the hypovolemic phase, the spleen was exteriorized and transected at the two ends of the organ as described earlier. We then returned the spleen to the abdominal cavity for uncontrolled bleeding. During this phase of uncontrolled haemorrhage, we closely observed the animal until a predetermined resuscitation-trigger point was reached. At that point, haemostasis was rapidly achieved by ligation of the splenic pedicle.
Intravenous resuscitation was initiated by returning the shed blood over $5 \mathrm{~min}$. Animals were then randomly allocated to receive fluid resuscitation with double the shed blood volume as Ringer's lactate [conventional resuscitation, CR]; or double the shed blood volume as Ringer's lactate containing either norepinephrine $(25 \mu \mathrm{g} / 100 \mathrm{~g})$, vasopressin ( 0.8 units/100g), lipid vesicles $(2.5 \mathrm{mM}$ lipid vesicles without ATP)-the lipid vesicles control group; or, lipid vesicles encapsulating ATP, that is, ATPv $(2.5 \mathrm{mM} / \mathrm{ml} \mathrm{lipid} / 2.5 \mathrm{mM}$ ATP). There were 10 animals in each group. We completed resuscitation in 30 minutes in all animals.

\section{Data reduction and statistics}

We performed descriptive statistics and presented data as mean and standard deviations for interval variables. Post-resuscitation survival times for all groups were computed from the survival curves generated by Prism-6 (GraphPad Software, Inc., La Jolla, CA, USA). The program uses the product-limit method of Kaplan and Meier to generate the survival curves, and the log-rank and the Gehan-Wilcoxon tests for comparisons between the survival curves. Survival data was presented and plotted as fractional survival, standard error as calculated by the Greenwood method, together with the $95 \%$ confidence interval. As the end-point measurement in each group is death, censored observations were not accounted for in the analysis of the survival curves. Differences in MAP and SI within the group and between groups were analyzed by two-way analysis of variance and Tukey's post-hoc test to correct for multiple comparisons using Prism-6 (GraphPad Software, Inc., La Jolla, CA, USA). A P-value of 0.05 (two-tailed) was considered as statistically significant.

\section{Results}

\section{Mean arterial pressure and shock index}

The Table 1 Shows the summary and statistical analysis of the mean arterial pressure (MAP) and shock index (SI) as determined for each of the five experimental groups at the baseline, after hemostasis, at $5 \mathrm{~min}$ after completion of resuscitation, and at the time of cardio-circulatory collapse.

MAP and SI at the pre-haemorrhage baseline and at the time-point of cardio-circulatory collapse were similar between individual animals within each group and between groups. Establishment of hemostasis after the second phase of uncontrolled haemorrhage produced similar mean arterial pressures and shock indices in individual animals within each group and between groups, suggesting a similar class of shock in all animals. By design, none of the five resuscitation modalities restored the MAP or the SI to the pre-haemorrhage levels. However, at 5 min time point following completion of resuscitation, the adjuvant ATPv resuscitation group maintained a significantly higher MAP when compared with the other 4 resuscitation groups.

\begin{tabular}{|l|l|l|l|l|l|l|}
\hline $\begin{array}{l}\text { Cardio- } \\
\text { dynamic } \\
\text { Index }\end{array}$ & HS/CR & $\begin{array}{l}\text { HS/CR+Lipid } \\
\text { Vesicles }\end{array}$ & HS/CR+Norepinephrine & HS/CR+Vasopressin & HS/CR+ATPv & P-value \\
\hline \multicolumn{2}{|l|}{ Mean Arterial Pressure (MAP) } \\
\hline 0 & $112.4 \pm 11.7$ & $111.0 \pm 14.0$ & $117.8 \pm 11.6$ & $117.4 \pm 17.0$ & $118.0 \pm 18.0$ \\
\hline
\end{tabular}


Citation: Zakaria ER, Joseph B, Jehan FS, Khan M, Algamal A, et al. (2017) Improved Post-Resuscitation Survival Time with Adjuvant Cytosolic Energy Replenishment in a Murine Model of Hemorrhagic Refractory Shock . J Surg Anesth 1: 110.

Page 4 of 7

\begin{tabular}{|c|c|c|c|c|c|c|}
\hline 1 & $41.5 \pm 8.5^{\mathrm{a}}$ & $47.1 \pm 8.3^{a}$ & $46.5 \pm 6.3^{a}$ & $43.2 \pm 7.8^{a}$ & $46.7 \pm 5.6$ & 0.35 \\
\hline 2 & $\begin{array}{|ll|}49.6 & \pm \\
17.8^{\mathrm{a}, \mathrm{b}} & \end{array}$ & $56.9 \pm 22.8$ & $54.2 \pm 19.6^{a, b}$ & $69.9 \pm 30.8^{a, b}$ & $76.2 \pm 15.1^{a, b}$ & 0.05 \\
\hline 3 & $\begin{array}{l}13.2 \\
12.9^{\mathrm{a}, \mathrm{b}, \mathrm{c}}\end{array}$ & $16.4 \pm 14.5^{\mathrm{a}, \mathrm{b}, \mathrm{c}}$ & $7.8 \pm 6.5^{\mathrm{a}, \mathrm{b}, \mathrm{c}}$ & $9.5 \pm 7.6^{a, b, c}$ & $14.8 \pm 9.0^{a, b, c}$ & 0.34 \\
\hline P-value & 0.001 & 0.001 & 0.001 & 0.001 & 0.001 & \\
\hline \multicolumn{7}{|c|}{ Shock Index (SI) } \\
\hline 0 & $2.8 \pm 0.3$ & $2.6 \pm 0.3$ & $2.5 \pm 0.22$ & $2.6 \pm 0.3$ & $2.5 \pm 0.2$ & 0.15 \\
\hline 1 & $4.7 \pm 1.2$ & $4.8 \pm 1.6$ & $3.9 \pm 0.6$ & $4.6 \pm 0.9$ & $3.9 \pm 0.8$ & 0.24 \\
\hline 2 & $4.5 \pm 0.9$ & $5.5 \pm 1.6$ & $4.2 \pm 1.5$ & $3.8 \pm 1.3$ & $3.5 \pm 0.6$ & 0.06 \\
\hline 3 & $\begin{array}{|ll|}13.2 & \pm \\
10.0^{\mathrm{a}, \mathrm{b}, \mathrm{c}} & \end{array}$ & $15.6 \pm 23.0^{\mathrm{a}, \mathrm{b}, \mathrm{c}}$ & $15.9 \pm 8.9^{a, b, c}$ & $16.5 \pm 12.7^{\mathrm{a}, \mathrm{b}, \mathrm{c}}$ & $10.5 \pm 4.8^{\mathrm{a}}$ & 0.84 \\
\hline$P$-value & 0.001 & 0.001 & 0.001 & 0.001 & 0.001 & \\
\hline
\end{tabular}

Table 1: Variations in MAP and SI within the group and among the groups.

Trends of the variations in the MAP and the SI over time from individual animals from each group are given in Figure 2.
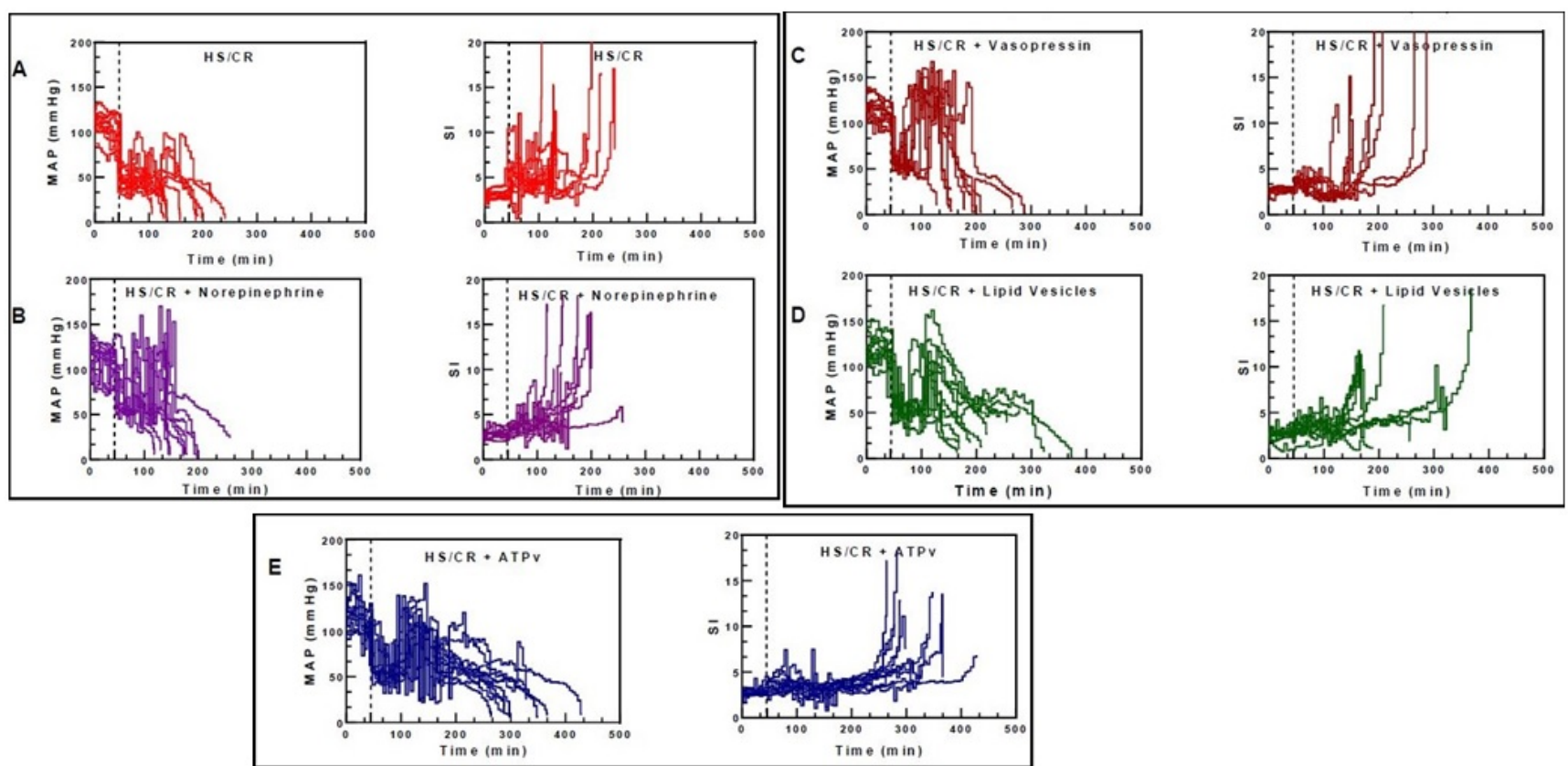

Figure 2: Trends of the mean arterial pressure (MAP) and the shock index (SI=Heart Rate/Systolic Pressure) from individual animals plotted as a function of time, respectively for each of the five resuscitation groups in: $\mathrm{A}=\mathrm{HS} / \mathrm{CR} ; \mathrm{B}=\mathrm{HS} / \mathrm{CR}+$ Norepinephrine; $\mathrm{C}=\mathrm{HS} / \mathrm{CR}+\mathrm{Vasopressin}$; $\mathrm{D}=\mathrm{HS} / \mathrm{CR}+\mathrm{Lipid}$ Vesicles without ATP; and in E=HS/CR+ATPv. HS=haemorrhagic shock (controlled haemorrhage by removal of $30 \%$ of the animals' blood volume +60 min of hypovolemia+uncontrolled haemorrhage); $\mathrm{CR}=$ Conventional Resuscitation (return of shed blood and $2 \mathrm{x}$ shed blood volume as lactated ringers). Lipid Vesicles=Lipid vesicles (no ATP); and ATPv=Lipid Vesicles encapsulating ATP; ATP=adenosine-5' -triphosphate; Hatched vertical line represents the time-point of haemorrhage initiation. HS=Haemorrhagic Shock (30\% removal of the blood volume+60 min of hypovolemia+uncontrolled bleeding until haemostasis). CR=Conventional Resuscitation (return of shed blood over 5 min followed by $2 \mathrm{x}$ the shed blood volume as ringer's lactate infused over the subsequent 25 min). All adjuvants were admixed with the ringers lactate crystalloid solution. 
As seen in the figure, MAP and SI varied considerably during the unsteady state of 25 min of infusion, much in accordance with the type of the adjuvant resuscitation used. Manual infusion of norepinephrine and vasopressin as guided by blood pressure monitoring, caused remarkable but transient spikes in blood pressure above the prehaemorrhage levels. However, both vasopressors failed to maintain the MAP after completion of resuscitation. This was associated with a steady decrease in MAP and increase of the SI until death from cardiocirculatory collapse. These trends contrast with the ATPv and the lipid vesicles groups. During the $25 \mathrm{~min}$ of the unsteady state of manual ATPv infusion and while observing for arterial pressure, ATPv caused transient decreases in both MAP and SI. When compared with the other 4 resuscitation groups, adjuvant $\mathrm{ATPv}$ maintained the MAP and the SI close to the baseline for a much longer time after completion of resuscitation. In addition, as seen in the figure 2 , a SI $\geq 4$ is a strong predictor for shock decompensation, whereas an abrupt increase in the SI to a ratio $>5$ suggests pending cardio-circulatory collapse and subsequent death from cardiac arrest.

\section{Post-resuscitation survival time}

Figure 3 depicts the survival fractions from the five resuscitation groups as computed by the Kaplan-Meier method.

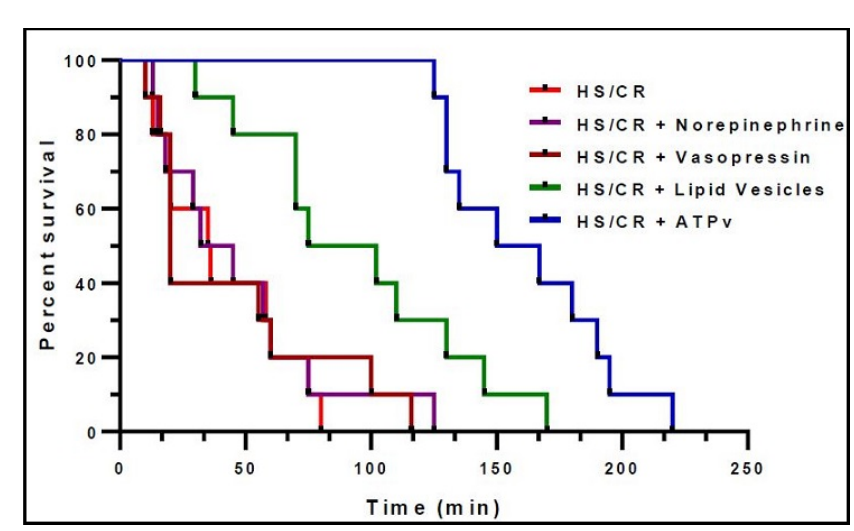

Figure 3: Kaplan Meier analysis of the survival curves. HS=Haemorrhagic Shock (30\% removal of the blood volume +60 min of hypovolemia+uncontrolled bleeding until haemostasis). $\mathrm{CR}=$ Conventional Resuscitation (return of shed blood over $5 \mathrm{~min}$ followed by $2 \mathrm{x}$ the shed blood volume as ringers lactate infused over the subsequent $25 \mathrm{~min}$ ). All adjuvants were admixed with the ringers lactate crystalloid solution; Groups are: HS/CR; HS/CR +Norepinephrine; HS/CR+Vasopressin; HS/CR+vesicles only and no ATP; HS/CR+ATPv; Lipid Vesicles=Lipid Vesicles (no ATP); and $\mathrm{ATPv}=$ Lipid Vesicles encapsulating ATP. ATP=adenosine-5 ${ }^{`}-$ triphosphate.

Comparison of the five survival curves using the log-rank test for trends rejected the null hypothesis in favor of significant difference between the five survival curves (Chi-square 35.64, $\mathrm{DF}=4, \mathrm{P}=0.0001$ ) and for trend (Chi-square $31.30, \mathrm{DF}=1, \mathrm{P}=0.0001$ ). The survival curve for the HS/CR group was similar to the curves of $\mathrm{HS} / \mathrm{CR}$ +Norepinephrine $(\mathrm{P}=0.131)$, and the HS/CR+Vasopressin $(\mathrm{P}=0.095)$, but significantly different from the curve of the HS/CR+Lipid Vesicles $(\mathrm{P}=0.0006)$. In contrast, the survival curve for the HS/CR+ATPv group was significantly different from the $\mathrm{HS} / \mathrm{CR}+$ Norepinephrine $(\mathrm{P}=0.00001), \mathrm{HS} / \mathrm{CR}+$ Vasopressin $(\mathrm{P}=0.00001)$, and the HS/CR+Lipid
Vesicles $(\mathrm{P}=0.0005)$. Level of significance for the log-rank (MantelCox) test was adjusted for multiple comparisons. Median postresuscitation times were $\mathrm{HS} / \mathrm{CR}$ (35.5 $\mathrm{min})$, HS/CR+Norepinephrine (38.5 min), HS/CR+Vasopressin (20 min), HS/CR+Lipid Vesicles (88.5 $\mathrm{min})$, and $\mathrm{HS} / \mathrm{CR}+\mathrm{ATPv}(158.5 \mathrm{~min})$.

\section{Discussion}

In this, prove of concept study, we reproduced refractory shock in rats to determine the efficacy and effectiveness of three resuscitation strategies in delaying cardio-circulatory arrest that follows this class of shock. Among the three resuscitation strategies evaluated, only the targeted cellular energy resuscitation strategy delayed cardiocirculatory arrest. Although, a modest increase in post-resuscitation survival time was obtained by resuscitation with the lipid vesicles lacking the encapsulated ATP, vasopressors exerted no postresuscitation survival benefits over conventional resuscitation with blood and crystalloids. Although our study is not a mechanistic study, interpretation of the outcome of each of the three targeted resuscitation strategies should take into consideration the pathogenesis of a refractory haemorrhagic shock and, in particular, hypovolemia. Lactated Ringer's is the most commonly used crystalloid in the resuscitation of haemorrhagic shock [22]. However, in progressive haemorrhagic hypovolemic shock in which bleeding is controlled, large crystalloid volumes are required to correct the volume deficit and to support the central hemodynamics. Such aggressive crystalloid resuscitation promotes hemodilution and coagulopathy, accelerates systemic inflammation, and usually fails to maintain blood flow and end-organ tissue perfusion [23-25]. The administration of vasopressors in shock due to etiologies other than hypovolemia is a recognized clinical practice [26]. In contrast, such therapy in hypovolemic shock does not achieve the therapeutic goals of restoring tissue perfusion and normalizing cellular metabolism.

In a previous study conducted in a pressure-controlled haemorrhagic shock model in rats, we demonstrated that cellular cytosolic energy replenishment restores and maintains the baseline pre-haemorrhage hemodynamic and blood flow to the gut without the need to correct for the vascular volume deficit with fluids [27]. These favorable outcomes were explained by the restoration of the energydependent cellular processes and by a strong positive inotropic action of ATP [28]. It is interesting to note that resuscitation with the lipid vesicles without the ATP maintained a higher post-resuscitation arterial pressure and produced a longer post-resuscitation survival time than the conventional or the pressure-support resuscitation with vasopressors. Although unexpected finding, there are several possible explanations for this observation. First, the composition of the lipid vesicles is similar to that of the cell membrane, as it was specifically designed to be unstable in order to fuse and integrate with cell membranes on contact. Because of these similarities, the integration of lipid vesicles within the cell membrane can potentially ameliorate the cell membrane structural abnormalities such as fluidity, which, as demonstrated in a number of studies, is known to occur with haemorrhage [29-31]. Second, the observed favorable effects of the lipid vesicles might relate to the ability of the phospholipid components of the vesicles to stimulate the $\mathrm{Na}+/ \mathrm{K}+-$ ATPase [32]. This helps to maintain resting membrane potential and cellular volume, which are altered by haemorrhage [30]. However, the exact mechanisms of the favorable effects of the lipid vesicles cannot be directly discerned from the present single outcome study. 
The vascular endothelium accounts for $\sim 24 \%$ of the total O2 consumption of the body [33] to generate energy from oxidative phosphorylation and glycolysis in order to support its multifaceted functions [34]. During conditions of energy substrate deprivation or depletion of cytosolic nucleotides as in traumatic haemorrhage, endothelial cells adapt by massive downregulation of their energydependent processes [35]. As a result, endothelial protein synthesis and the ability to control and maintain vascular tone and myogenic response are severely impaired. In our study, cellular cytosolic energy replenishments were restricted to the duration of the ATPv infusion. It is likely that the rate of cytosolic ATP replenishment in our current study was below the threshold required to fulfil the continued cellular energy needs. Therefore, it remains to be determined if continuous cytosolic ATP replenishment would completely prevent cardiocirculatory collapse. This is beyond our current proving of concept study.

We conducted preliminary studies using vasopressor concentrations that were previously published [36-38]. These concentrations even at prolonged and slow infusion times caused very high spikes in blood pressure and remarkably prolonged resuscitation time. It was also observed that a number of animals developed hydrostatic pulmonary edema and had no improvement in post-resuscitation survival time. Therefore, in the experiments summarized in the present study, the doses of norepinephrine and vasopressin were reduced by $50 \%$. Still, these doses did not protect against cardio-circulatory collapse to improve post-resuscitation survival time. In contrast, the ATPv formula used in our present study contained free ATP in the medium, which caused transient drop of blood pressure that lasted for seconds during the resuscitation period. These drops in blood pressure were not sustained as the half-life of free ATP in the blood is less than $40 \mathrm{~s}$. Nevertheless, to overcome any significant effect on blood pressure, the administration of norepinephrine, vasopressin, or ATPv has to be administered manually as small boluses and not as continuous infusion. While continuously watching the blood pressure, this manual infusion technique allows for the blood pressure to return to baseline before the next bolus is infused. We should emphasize that all animals in our current study received the same class of a refractory shock, equivalent blood and crystalloid volumes, and similar resuscitation times. We acknowledged that the standard of care resuscitation of this class of refractory shock is to evoke the modern massive transfusion protocols, and therefore all the animals in the present study are underresuscitated by design. This should not negate, refute or challenge the significant difference in post-resuscitation survival times between the five groups.

\section{Conclusions}

We concluded that the replenishment of the depleted cellular cytosolic energy stores in a refractory haemorrhagic hypotensive shock prolongs post-resuscitation survival time and delays cardio-circulatory arrest. This buys time for the initiation of definitive resuscitation protocols. Cellular energy failure appears to contribute to the pathogenesis of shock refractoriness to resuscitation efforts. The temporary administration of vasopressors for pressure-support resuscitation of a refractory haemorrhagic hypovolemic shock exerts no survival benefits.

MAP and SI presented as mean \pm SD. HS=haemorrhagic shock (30\% removal of the blood volume $+60 \mathrm{~min}$ of hypovolemia +uncontrolled bleeding until haemostasis). $\mathrm{CR}=$ Conventional Resuscitation (return of shed blood over 5 min followed by $2 \mathrm{x}$ the shed blood volume as ringer's lactate infused over the subsequent $25 \mathrm{~min}$ ). All adjuvants were admixed with the ringers lactate crystalloid solution.

ATPv=Lipid Vesicles encapsulating ATP; ATP=adenosine-5 triphosphate; $0,1,2$, and 3 were measurements at baseline, immediately after haemostasis (ligation of splenic pedicle), $5 \mathrm{~min}$ after completion of resuscitation, and at the time of cardio-circulatory collapse, respectively. Variation within the group's time points $1 \mathrm{vs} .0$ (a) $\mathrm{p}<0.001 ; 2$ vs. 1 (b) $\mathrm{p}<0.001$; and 3 vs. 2 (c) $\mathrm{p}<0.001$ by two-way analysis of variance and Tukey's multiple comparison post hoc test. Vesicles encapsulating ATP; ATP=adenosine-5 ${ }^{`}$-triphosphate.

\section{References}

1. Rhee P, Joseph B, Pandit V, Aziz H, Vercruysse G, et al. (2014) Increasing trauma deaths in the United States. Ann Surg 260: 13-21.

2. Kauvar DS, Lefering R, and Wade CE (2006) Impact of hemorrhage on trauma outcome: an overview of epidemiology, clinical presentations, and therapeutic considerations. J Trauma 60: S3-11.

3. Sauaia A, Moore FA, Moore EE, Moser KS, Brennan R, et al. (1995) Epidemiology of trauma deaths: a reassessment. J Trauma 38: 185-193.

4. Passos E, Dingley B, Smith A, Engels PT, Ball CG, et al. (2014) Tourniquet use for peripheral vascular injuries in the civilian setting. Injury 45 : 573-577.

5. Zietlow JM, Zietlow SP, Morris DS, Berns KS, Jenkins DH (2015) Prehospital Use of Hemostatic Bandages and Tourniquets: Translation From Military Experience to Implementation in Civilian Trauma Care. J Spec Oper Med 15: 48-53.

6. Holcomb JB, Jenkins D, Rhee P, Johannigman J, Mahoney P, et al. (2007) Damage control resuscitation: directly addressing the early coagulopathy of trauma. J Trauma 62: 307-310.

7. Holcomb JB, Tilley BC, Baraniuk S, Fox EE, Wade CE, et al. (2015) Transfusion of plasma, platelets, and red blood cells in a $1: 1: 1$ vs a $1: 1: 2$ ratio and mortality in patients with severe trauma: the PROPPR randomized clinical trial. JAMA 313: 471-482.

8. Langan NR, Eckert M, Martin MJ (2014) Changing patterns of inhospital deaths following implementation of damage control resuscitation practices in US forward military treatment facilities. JAMA Surg 149: 904-912.

9. Vincent JL, De BD (2013) Circulatory shock. N Engl J Med 369: 1726-1734.

10. Chaudry IH, Planer GJ, Sayeed MM, Baue AE (1973) ATP depletion and replenishment in hemorrhagic shock. Surg Forum 24: 77-79.

11. Chaudry IH, Sayeed MM, Baue AE (1974) Effect of adenosine triphosphate-magnesium chloride administration in shock. Surgery 75 : 220-227.

12. Chaudry IH, Sayeed MM, Baue AE (1974) Depletion and restoration of tissue ATP in hemorrhagic shock. Arch Surg 108: 208-211.

13. Zambon A, Waxman K, Daughters K, Eloi L (1994) ATP-MgCl2 added to resuscitation improves survival in an experimental model of hemorrhagic shock. Resuscitation 28: 253-257.

14. Chaudry IH, Sayeed MM, Baue AE (1976) Alterations in high-energy phosphates in hemorrhagic shock as related to tissue and organ function. Surgery 79: 666-668.

15. Van Way CW, Dhar A, Reddy R, Evans L, Wogahn B, et al. (1996) Changes in adenine nucleotides during hemorrhagic shock and reperfusion. J Surg Res 66: 159-166.

16. Zakaria ER, Matheson PJ, Flessner MF, Garrison RN (2008) Hemorrhagic shock and resuscitation-mediated tissue water distribution is normalized by adjunctive peritoneal resuscitation. J Am Coll Surg 206: 970-980.

17. Ehringer WD, Niu W, Chiang B, Wang OL, Gordon L, et al. (2000) Membrane permeability of fructose-1,6-diphosphate in lipid vesicles and endothelial cells. Mol Cell Biochem 210: 35-45. 
Citation: Zakaria ER, Joseph B, Jehan FS, Khan M, Algamal A, et al. (2017) Improved Post-Resuscitation Survival Time with Adjuvant Cytosolic Energy Replenishment in a Murine Model of Hemorrhagic Refractory Shock . J Surg Anesth 1: 110.

Page 7 of 7

18. Ehringer WD, Su S, Chiangb B, Stillwell W, Chien S (2002) Destabilizing effects of fructose-1,6-bisphosphate on membrane bilayers. Lipids 37: 885-892.

19. Zakaria ER, Ehringer WD, Tsakadze N, Li N, Garrison RN (2005) Direct energy delivery improves tissue perfusion after resuscitated shock. Surgery 138: 195-203.

20. Lee HB, Blaufox MD (1985) Blood volume in the rat. J Nucl Med 26 72-76.

21. Schafer K, Van SC, Hinojosa-Laborde C, Convertino VA (2013) Physiologic mechanisms underlying the failure of the "shock index" as a tool for accurate assessment of patient status during progressive simulated hemorrhage. J Trauma Acute Care Surg 75: S197-S202.

22. Krausz MM (2006) Initial resuscitation of hemorrhagic shock. World J Emerg Surg 1: 14.

23. Hierholzer C, Harbrecht B, Menezes JM, Kane J, MacMicking J, et al. (1998) Essential role of induced nitric oxide in the initiation of the inflammatory response after hemorrhagic shock. J Exp Med 187: 917-928.

24. Rhee P, Burris D, Kaufmann C, Pikoulis M, Austin B, et al. (1998) Lactated Ringer's solution resuscitation causes neutrophil activation after hemorrhagic shock. J Trauma 44: 313-319.

25. Zakaria ER, Spain DA, Harris PD, Garrison RN (2002) Resuscitation regimens for hemorrhagic shock must contain blood. Shock 18: 567-573.

26. Hollenberg SM (2011) Vasoactive drugs in circulatory shock. Am J Respir Crit Care Med 183: 847-855.

27. Zakaria ER, Ehringer WD, Tsakadze N, Li N, Garrison RN (2005) Direct energy delivery improves tissue perfusion after resuscitated shock. Surgery 138: 195-203.

28. Regnier M, Rivera AJ, Chen Y, Chase PB (2000) 2-deoxy-ATP enhances contractility of rat cardiac muscle. Circ Res 86: 1211-1217.

29. Maslova MN, Kazennov AM, Katiukhin LN, Novozhilov AV, Skverchinskaia EA, et al. (2007) Changes of physiological and biochemical characteristics of rat erythrocytes after blood loss. Zh Evol Biokhim Fiziol 43: 414-418.

30. Shires GT, Cunningham JN, Backer CR, Reeder SF, Illner H, et al. (1972) Alterations in cellular membrane function during hemorrhagic shock in primates. Ann Surg 176: 288-295

31. Zaets SB, Berezina TL, Morgan C, Kamiyama M, Spolarics Z, et al. (2003) Effect of trauma-hemorrhagic shock on red blood cell deformability and shape. Shock 19: 268-273.

32. Haviv H, Habeck M, Kanai R, Toyoshima C, Karlish SJ (2013) Neutral phospholipids stimulate Na,K-ATPase activity: a specific lipid-protein interaction. J Biol Chem 288: 10073-10081.

33. Tsai AG, Johnson PC, Intaglietta M (2003) Oxygen gradients in the microcirculation. Physiol Rev 83: 933-963.

34. Culic O, Gruwel ML, Schrader J (1997) Energy turnover of vascular endothelial cells. Am J Physiol 273: C205-C213.

35. Culic O, Decking UK, Schrader J (1999) Metabolic adaptation of endothelial cells to substrate deprivation. Am J Physiol 276: C1061C1068.

36. Liu L, Tian K, Xue M, Zhu Y, Lan D, et al. (2013) Small doses of arginine vasopressin in combination with norepinephrine "buy" time for definitive treatment for uncontrolled hemorrhagic shock in rats. Shock 40: 398-406.

37. Meybohm P, Cavus E, Bein B, Steinfath M, Weber B, et al. (2007) Small volume resuscitation: a randomized controlled trial with either norepinephrine or vasopressin during severe hemorrhage. J Trauma 62: 640-646.

38. Poloujadoff MP, Borron SW, Amathieu R, Favret F, Camara MS, et al. (2007) Improved survival after resuscitation with norepinephrine in a murine model of uncontrolled hemorrhagic shock. Anesthesiology 107: 591-596. 